\title{
Finite element small-signal simulation of electromagnetic devices considering eddy currents in the laminations
}

\author{
Omar Bottesi, Luigi Alberti, Ruth V. Sabariego and Johan Gyselinck
}

\begin{abstract}
This paper presents the validation of a small-signal finite element simulation strategy comparing different simulation approaches. At first a full 3D non-linear model in the time domain is presented. Then the small-signal strategy has been applied to the 3D model achieving a considerable reduction of computational time. In both $3 \mathrm{D}$ models, the eddy currents in the laminations are fully modelled. Then, in order to avoid 3D model, a 2D model has been considered. In this case, eddy currents are included adopting a homogenization technique. In the $2 \mathrm{D}$ case, both non-linear time domain simulations and smallsignal strategy have been implemented.

The results of the four considered simulation strategies are in very good agreement, proving that the smallsignal simulation strategy can be profitably used in order to reduce significantly the computational cost. As an example, the paper considers a two port mutual inductor as a test model.
\end{abstract}

Index Terms - finite element method, small-signal, frozen permeability, eddy currents, homogenization

\section{INTRODUCTION}

Nowadays, finite element (FE) simulations play an important role in the analysis of electromagnetic devices, thanks to their capability to include in the analysis various aspects such as complex domain shapes and nonlinear characteristics of materials. Different techniques have been developed to consider both static and dynamic fields, for example considering eddy current problems and also considering movement [1], [2]. The interest in the inclusion of parasitic phenomenon in electrical devices is increased in the last years. This has been also driven by new efficiency standards that force manufacturers in designing more efficient electrical system in order to reduce global emissions and increase the global sustainability.

Among the others, eddy currents in the laminations have been investigated considering different approaches [3]-[5]. When the precise inclusion of eddy current is required, normally a 3D model has to be considered. This increase

\footnotetext{
Omar Bottesi is with the Faculty of Science and Technology, Free University of Bozen-Bolzano, Bolzano (Italy), omar.bottesi@natec.unibz.it.

Luigi Alberti is with the Dept. of Industrial Engineering, University of Padova, Padova (Italy), luigi.alberti@unipd.it.

Ruth Sabariego is with the Dept. of Electrical Engineering, Katholieke Universiteit Leuven, Leuven, Belgium, ruth.sabariego@esat.kuleuven.be.

Johan Gyselinck is with the BEAMS Departement, Université libre de Bruxelles, Bruxelles, Belgium, johan.gyselinck@ulb.ac.be
}

significantly the computational costs of the simulation. Alternative approaches have been proposed where endeffects of eddy currents are typically neglected and where the domain under investigation can be scaled down to $2 \mathrm{D}$ models [6].

Another topic in which there is an increasing development, is the characterization of the system parameters including parasitic effects. Such a topic is not directly related to the computation of the system losses and to the increasing of overall efficiency as described above. Often the system parameters are required in order to tune the controller and to fulfill the design requirements such as dynamic response. As an example, the inclusion of eddy currents in the lamination for the computation of the incremental parameters of an electrical machine in order to determine the rotor position has been recently proposed [7], [8]. The inclusion of the parasitic effects during the computation of the system parameters, allows to evaluate their impact on the parameters themselves. In this way a more precise parameter computation can be performed [9]. On the other hands, parasitic phenomenon can also be exploited to shape the machine parameters, so that the machine performance results improved. As an example, in [10] eddy currents induced in the permanent magnets of a synchronous machine are exploited to create a high frequency resistive saliency of the machine useful for sensorless control.

In the latter case, the system is supplied with two frequencies: the main excitation (for example the torqueproducing current of the motor) and a high frequency signal (a carrier signal to identify the rotor position). In such a case the system can be profitably simulated using a small-signal approach [7] that relies on the frozen permeability method. Such a method assumes that the two frequencies do not interact significantly for the parasitic effects. This, of course, is not true in general but it is a good approximation which gives satisfactory results in many practical applications, for example when the main excitation is at very low frequency (or even constant terms, i.e. DC values). This is the case of electrical machines operating at very low speed where signal injection is used for tracking the rotor position.

In this paper the small-signal FE simulation strategy is validated comparing different simulation approaches. At first full 3D nonlinear simulations in the time domain are presented. In such a case the eddy current in the 
laminations are fully modeled and this case is considered as a reference for the further simulations. Then the smallsignal strategy has been applied to the 3D model achieving a considerable reduction of computational time. Also in this case the eddy currents in the laminations are fully modeled. In order to avoid 3D model, a 2D model has been considered. In this case, eddy currents are included adopting a homogenization technique as reported in [11]. In such a case, end effects of eddy currents are neglected. Such an approximation has been found well acceptable in most situations. In the 2D case, both nonlinear time domain simulations and small-signal strategy have been implemented.

The four considered simulation strategies yield results in very good agreement, proving that the small-signal simulation strategy can be profitably used in order to reduce significantly the computational cost. As an example, the paper considers a two port mutual inductor as a test model.

\section{Description of the test Model}

In this Section the electromagnetic test model adopted in the computation is described. It consists of a two port mutual inductor with a nonlinear laminated core as shown in Fig. 1. Such test model is here considered for the sake of simplicity to better clarify the proposed approach. Nevertheless, the simulation strategy described in the following can be straightforwardly extended to consider any electromagnetic device with more phases and ports.

The goal of the simulation is to model properly the voltage-current relation at the system terminals considering the nonlinear characteristic of the laminated iron core and the eddy currents induced in the laminations by the time varying flux density.

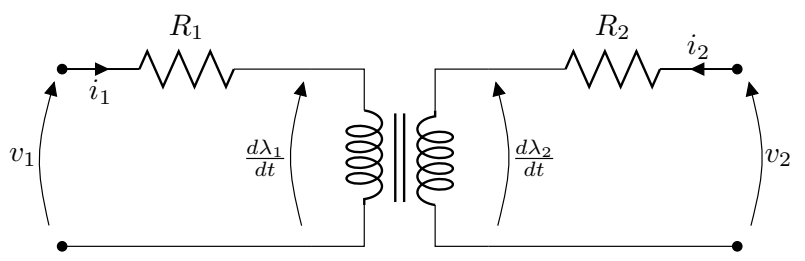

Fig. 1. Two port mutual inductor

Assuming the currents $i_{1}, i_{2}$ in the two coils as state variables, the voltage equations at the terminals can be written as:

$$
\left\{\begin{array}{l}
v_{1}=R_{1} i_{1}+\frac{d \lambda_{1}\left(i_{1}, i_{2}\right)}{d t} \\
v_{2}=R_{2} i_{2}+\frac{d \lambda_{2}\left(i_{1}, i_{2}\right)}{d t}
\end{array}\right.
$$

where $R_{1}$ and $R_{2}$ are the resistances of the two windings. The dependence of the flux linkages on both the currents due to the saturation has been explicitly highlighted.

\section{A. Small-signal model}

The electrical behavior of the device is completely described by (1) the integration of which is not always possible in a closed form due to the strong nonlinearity. Nevertheless, in many applications it is of great interest to characterize the system at a specific operating point considering only small variations around the working point and not the general system response. For example it could be interesting to compute the incremental parameters (both inductances and resistances) at the electrical ports for different operating currents. In such a case a smallsignal model can be adopted, i.e. the currents and the flux linkages can be written as:

$$
\begin{gathered}
\left\{\begin{array}{l}
i_{1}=I_{1}+\delta i_{1} \\
i_{2}=I_{2}+\delta i_{2}
\end{array}\right. \\
\left\{\begin{array}{l}
\lambda_{1}=\Lambda_{1}+\delta \lambda_{1} \\
\lambda_{2}=\Lambda_{2}+\delta \lambda_{2}
\end{array}\right.
\end{gathered}
$$

where $I_{1}, I_{2}$ are the currents at the actual operating point, $\Lambda_{1}$ and $\Lambda_{2}$ are the corresponding flux linkages. The current variations $\left(\delta i_{1}, \delta i_{2}\right)$ produce a corresponding variation in the flux linkages $\delta \lambda_{1}$ and $\delta \lambda_{2}$ that depend on both currents and can be expressed as:

$$
\left\{\begin{array}{l}
\delta \lambda_{1}=\frac{\partial \lambda_{1}}{\partial i_{1}} \delta i_{1}+\frac{\partial \lambda_{1}}{\partial i_{2}} \delta i_{2}=l_{11} \delta i_{1}+m_{12} \delta i_{2} \\
\delta \lambda_{2}=\frac{\partial \lambda_{2}}{\partial i_{1}} \delta i_{1}+\frac{\partial \lambda_{2}}{\partial i_{2}} \delta i_{2}=m_{21} \delta i_{1}+l_{22} \delta i_{2}
\end{array}\right.
$$

$l_{11}, m_{12}, m_{21}$ and $l_{22}$ are the incremental inductances seen at the device terminals, defined as the partial derivative of the flux linkage evaluated in the considered working point $\left(I_{1}, I_{2}\right)$. For the mutual inductances it results $m_{12}=m_{21}$ due to the reciprocity theorem. Using (1) the small-signal variation of the terminal voltages can be expressed as:

$$
\left\{\begin{array}{l}
\delta v_{1}=R_{1} \delta i_{1}+l_{11} \frac{d}{d t} \delta i_{1}+m_{12} \frac{d}{d t} \delta i_{2} \\
\delta v_{2}=R_{2} \delta i_{2}+m_{21} \frac{d}{d t} \delta i_{1}+l_{22} \frac{d}{d t} \delta i_{2}
\end{array}\right.
$$

\section{B. Sinusoidal variation}

Among the various time variations, sinusoidal excitation is one of the most important in practical applications. In such a case, complex notation can be adopted, and (5) can be expressed as:

$$
\left\{\begin{array}{l}
\bar{v}_{1}=R_{1} \bar{\imath}_{1}+j \omega l_{11} \bar{\imath}_{1}+j \omega m_{12} \bar{\imath}_{2} \\
\bar{v}_{2}=R_{2} \bar{\imath}_{2}+j \omega m_{21} \bar{\imath}_{1}+j \omega l_{22} \bar{\imath}_{2}
\end{array}\right.
$$

where overlined symbols are used for phasors and $\omega=2 \pi f$ is the angular frequency of the sinusoidal excitation.

In order to take into account also parasitic phenomena such as eddy currents in the laminations, (6) can be generalized adopting impedances instead of inductances. Therefore, when eddy currents are taken into account, (6) can be expressed as:

$$
\left\{\begin{array}{l}
\bar{v}_{1}=R_{1} \bar{\imath}_{1}+\dot{z}_{11} \bar{\imath}_{1}+\dot{z}_{12} \bar{\imath}_{2} \\
\bar{v}_{2}=R_{2} \bar{\imath}_{2}+\dot{z}_{21} \bar{\imath}_{1}+\dot{z}_{22} \bar{\imath}_{2}
\end{array}\right.
$$

The impedances $\dot{z}_{11}, \dot{z}_{12}, \dot{z}_{21}$ and $\dot{z}_{22}$ take into consideration either the resistive or inductive effects of the small variation. 


\section{Finite element simulations}

In this Section the FE simulation strategies are described. As reference model, 3D nonlinear time domain magnetodynamic simulations have considered including eddy currents in the laminations. As a benefit, eddy currents are fully modeled within each lamination considering end effects. On the other hand, this kind of model requires very large computational costs, both in terms of time and memory. Moreover, drawing the geometry and imposing the constrains can be rather complicated.

In order to avoid 3D drawbacks, a 2D geometry has been considered. Then, eddy currents have been included applying a nonlinear homogenization technique [11]. It is based on a 1D model of the lamination coupled with the FE equations to approximate the field distribution in the lamination. The eddy currents are approximated with a II-order homogenization which is accurate enough for the considered frequencies and lamination thickness. In addition, the 2D geometry exhibits several advantages: the complexity of the geometry is reduced and the constraints imposition is easier. As a drawback, the end effects have to be neglected.

However, the computational time remain rather large when time domain simulations are carried out. To reduce significantly such a computational cost, the small-signal simulation strategy can be profitably adopted. It consists in adopting the frozen permeability technique avoiding transient simulations. The frozen-permeability technique has been used for different purposes: modeling of electrical machines under saturation [12], evaluation of the average torque considering the flux linkage contributions [13], isolation of the torque components [14]. For those purposes, only a magnetostatic analysis is required. Nevertheless, this technique can be applied to magnetodynamic analysis taking into account the parasitic effects such as the eddy currents in the lamination. This application can be considered as a new technique for the determination of the incremental parameters and it will be thus fully described in the next sections highlighting how the computational time is further reduced.

Moreover, the equations of the external circuits of $2 \mathrm{D}$ and $3 \mathrm{D}$ models have been included in the analysis [15] and, as a result, both current and voltage sources can be applied.

To make the comparison as complete as possible, both $2 \mathrm{D}$ and 3D models have been tested adopting both time domain formulation and the small-signal technique with frozen permeability. Further details are given in following sections.

\section{A. Time domain formulation}

In this Subsection the time domain formulation is considered as described in [1], [2]. The supply sources can be directly applied and they are composed by a bias component plus a sinusoidal small-signal as follow:

$$
\left\{\begin{array}{l}
i_{1}(t)=I_{1}+\hat{i}_{1} \sin \left(2 \pi f t+\alpha_{1}\right) \\
i_{2}(t)=I_{2}+\hat{i}_{2} \sin \left(2 \pi f t+\alpha_{2}\right)
\end{array}\right.
$$

where $I_{1}$ and $I_{2}$ are the bias current components which produce the main magnetization of the system, $\hat{i}_{1}$ and $\hat{i}_{2}$ the sinusoidal small-signal currents superimposed to the bias components, while $\alpha_{1}$ and $\alpha_{2}$ are the shift displacement of the small-signal waveforms. Since the $\hat{i}_{1}$ and $\hat{i}_{2}$ are small-signals the ratio between the injection signals and the bias components is assumed less than $5 \%$, otherwise, nonlinear phenomena can introduce error in the computation of the parameters described in (7). More details on this hypothesis are reported in the next sections.

The number of the simulated periods have to be high enough (e.g. 8 periods) so that the system can reach steady state. In order to compute the incremental parameters, two simulations have to be carried out, one at each port As a result, two complete transients must be solved. The incremental parameters are computed taking into account only the last period at steady-state.

Alternatively, the simulation can be voltage driven by using external circuits [15].

\section{B. Small-signal technique with frozen permeability}

As mentioned above, frozen permeability method allows to compute the incremental parameters of the device when the machine is saturated and to take into account the parasitic phenomena such as eddy currents due to the high frequency injection. This aspect can be particularly advantageous either for the determination of the incremental parameters or for reducing the computational time.

The proposed simulation strategy is composed of:

1) bias current simulation: a nonlinear magnetostatic simulation is performed at a specific working point $\left(I_{1}, I_{2}\right)[1],[2]$. This allows to consider the nonlinearity of the iron and all the sources in the machines, i.e. winding currents and permanent magnet (if any). The computed magnetic flux density $\mathbf{B}_{0}$ of each element of the mesh is stored for the next step.

2) reluctivity tensor construction: from the stored $\mathbf{B}_{0}$, the differential reluctivity tensor for the smallsignal simulations is computed. A tensor is considered in order to represent the iron reluctivity since the magnetic field is, in general, not aligned with the main field [16]. Adopting the 2D Cartesian coordinates it can be written as:

$$
\begin{aligned}
{\left[\begin{array}{ll}
\frac{\partial h_{x}}{\partial b_{x}} & \frac{\partial h_{x}}{\partial b_{y}} \\
\frac{\partial h_{y}}{\partial b_{x}} & \frac{\partial h_{y}}{\partial b_{y}}
\end{array}\right] } & =\nu\left(\mathbf{B}_{0}\right)\left[\begin{array}{ll}
1 & 0 \\
0 & 1
\end{array}\right] \\
& +\left.2 \frac{d \nu}{d \mathbf{B}^{2}}\right|_{\mathbf{B}_{0}}\left[\begin{array}{ll}
\partial b_{x} \partial b_{x} & \partial b_{x} \partial b_{y} \\
\partial b_{y} \partial b_{x} & \partial b_{y} \partial b_{y}
\end{array}\right]
\end{aligned}
$$

where the scalar reluctivity $\nu\left(\mathbf{B}_{0}\right)$ is a single-valued function, and $\left.\frac{d \nu}{d \mathbf{B}^{2}}\right|_{\mathbf{B}_{0}}$ is the derivative of the reluctivity respect to the flux density square evaluated at the stored valued $\mathbf{B}_{0}$.

3) Small-signal simulation: then, time-harmonic simulation are carried out. In particular, considering different values of the injection frequency $f$. The reluctivity tensor is considered in the iron. This 
simulation step is characterized by small variations around the working point, only the injection signal are imposed during the simulation. Since sinusoidal quantities are considered, complex voltages, currents and flux linkages can be adopted. For the examined case, two current driven simulations are required supplying each port of the two port mutual inductor separately in order to compute the parameters $\dot{z}_{11}$, $\dot{z}_{12}=\dot{z}_{21}$ and $\dot{z}_{22}$. The computation of the incremental parameters $\dot{z}_{11}, \dot{z}_{12}=\dot{z}_{21}$ and $\dot{z}_{22}$ may thus be easily carried out as following:

$$
\begin{array}{ll}
\dot{z}_{11}=\left.\frac{\bar{v}_{1}}{\bar{\imath}_{1}}\right|_{\bar{\imath}_{2}=0} \quad \dot{z}_{12}=\left.\frac{\bar{v}_{1}}{\bar{\imath}_{2}}\right|_{\bar{\imath}_{1}=0} \\
\dot{z}_{21}=\left.\frac{\bar{v}_{2}}{\bar{\imath}_{1}}\right|_{\bar{\imath}_{2}=0} \quad \dot{z}_{22}=\left.\frac{\bar{v}_{2}}{\bar{v}_{2}}\right|_{\bar{\imath}_{1}=0}
\end{array}
$$

Generally, the number of magnetodynamic simulations are as many as the number of the device ports.

In the case of the two port mutual inductor, the simulations have been carried out supplying the device with bias currents at both terminals and, afterwards, injecting a high frequency signal, first, at the primary terminal and, then, at the secondary terminal. Fig. 2 helps to understand the simulation procedure.

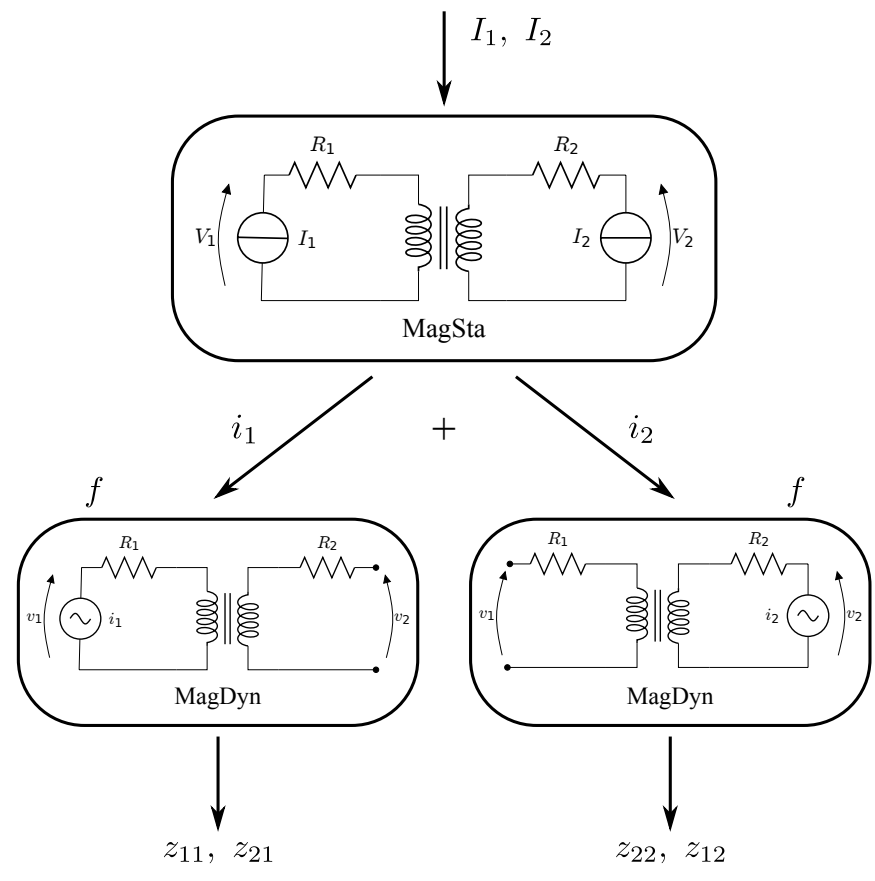

Fig. 2. Example of the simulation strategy. The label "MagSta" indicates the magnetostatic simulation, while "MagDyn" indicates the magnetodynamic simulation.

The simulation steps can be fully repeated in different operating conditions in order to characterize the device. Moreover, if the operating conditions are fixed, just one bias current simulation can be carried out. Then, in such an operating condition, it is possible to perform as many small-signal simulations as necessary in order to characterize the device, e.g. varying the injection frequency or the injection current. As a result, a reduction of the computational time is obtained since the bias current simulation requires the main computational time due to the consideration of the material nonlinearity.

\section{Application EXAmple}

In order to validate the simulation strategy, 2D and $3 \mathrm{D}$ simple models of the two port mutual inductor have been simulated. A sketch of the geometry is shown in Fig. 3. The $2 \mathrm{D}$ geometry is drawn in the $x y$-plane and it is composed by a iron stack laminated along $z$. Two stranded coils are wound around the vertical legs of the iron stack. The inductor is surrounded by air bounded by a line where a Dirichlet boundary condition is imposed. The 3D geometry has been obtained by extruding the $2 \mathrm{D}$ geometry along the thickness of lamination. In order to reduce the computational costs, only one lamination is considered. Fig. 4 shows a detail of the 3D lamination. The surface and the thickness discretization is highlighted. In particular, nine layers along the lamination thickness (uniform) are taken into account and prismatic mesh is considered in order to keep the same mesh for all the layers. The supply sources, both current and voltage, are applied by means of external circuit coupling. The main data of the model are reported in Tab. I.

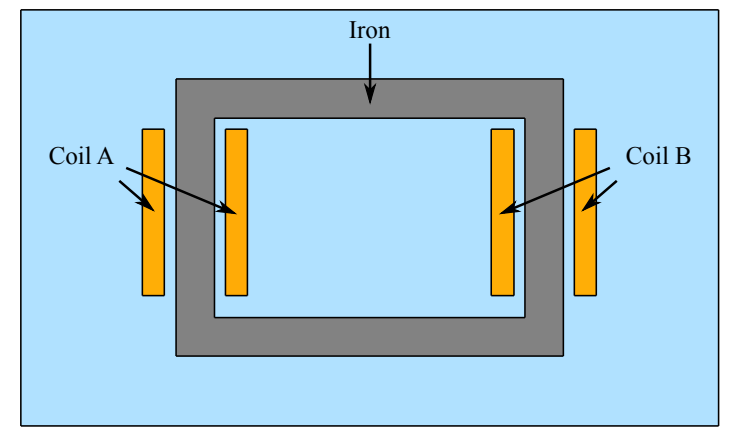

Fig. 3. Cross-section of the two port mutual inductor geometry (2D model)

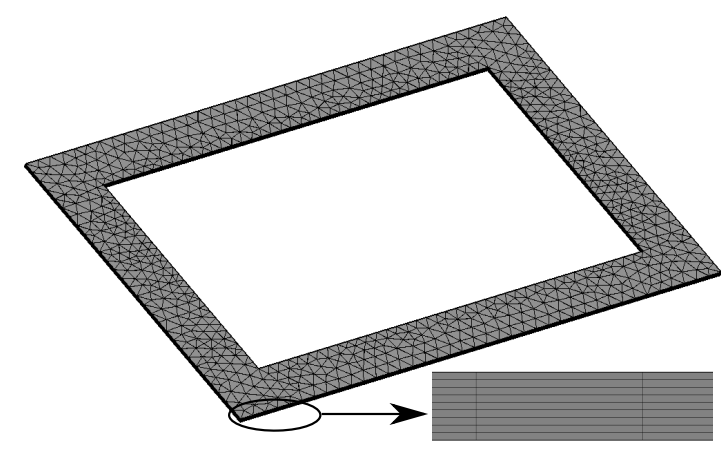

Fig. 4. Mesh of the lamination in the 3D model. Detail of the layers used to discretize the lamination along $\mathrm{z}$. 
TABLE I

MAIN GEOMETRICAL AND MATERIAL DATA OF THE TWO PORT MUTUAL INDUCTOR

\begin{tabular}{llll}
\hline Winding turns & $N_{1}, N_{2}$ & 1000 & \\
lamination height & $h_{\text {iron }}$ & 50 & $\mathrm{~mm}$ \\
lamination width & $w_{\text {iron }}$ & 70 & $\mathrm{~mm}$ \\
yoke thickness & $t k_{\text {iron }}$ & 7 & $\mathrm{~mm}$ \\
lamination thickness & $d$ & 0.5 & $\mathrm{~mm}$ \\
iron conductivity & $\sigma_{f e}$ & $10^{6}$ & $\mathrm{~S} / \mathrm{m}$ \\
\hline
\end{tabular}

A. $2 D$ and $3 D$ simulations comparing small-signal and time domain techniques

This Section reports the comparison among the simulation results obtained with the different simulation techniques. For such a comparison, different aspects have to be considered. In particular, the following aspects will be fully addressed:

- influence of small-signal perturbation;

- global quantities computation;

- computational costs.

In the following, the performed simulations are denoted as:

1) 3D-TD: 3D time domain formulation with explicit inclusion of eddy currents;

2) 3D-SS: 3D formulation considering small-signal technique and explicit inclusion of eddy currents;

3) 2D-TD: 2D time domain formulation applying homogenization technique for computing the eddy currents in the lamination;

4) 2D-SS: 2D formulation considering small-signal technique and homogenization technique for computing the eddy currents in the lamination.

1) Influence of small-signal perturbation: before comparing the simulation results, it is important to investigate the validity range for the small-signal technique. As an assumption of the small-signal method, the high frequency currents or voltages have to produce a linear variation of the field around the steady state working point. If the amplitude of high frequency signal is too large with respect the nominal current of the device, it can introduce nonlinear phenomena which can compromise the simulation results. Therefore, it is important to determine the maximum amplitude of the high frequency signals in order to avoid nonlinear phenomena. This can be obtain comparing time domain and small-signal simulations by fixing the bias component and changing the amplitude of the high frequency signal. For such an investigation, the system has been considered in a saturated condition and different percentages of the ratio between the high frequency signal and the bias component $\hat{i}_{1} / I_{1}$ are considered, i.e. $5 \%, 10 \%, 20 \%, 30 \%, 40 \%$. Being the bias current $I_{1}$ is fixed at $2 \mathrm{~A}$, the high frequency currents results in 0.1 , $0.2,0.4,0.6$ and $0.8 \mathrm{~A}$. Moreover, in this case the currents at the second port, i.e. the bias current $I_{2}$ and the high frequency current $\hat{i}_{2}$, are assumed equal to zero. Only 2D time domain simulations have been considered.

Tab. II reports the iron losses due to the eddy currents in the lamination for the considered simulation cases. The label $P_{l a m-T D}$ represents the iron losses in time domain, while $P_{l a m-S S}$ represents the iron losses computed by small-signal technique. The iron losses are expressed in p.u. with respect the losses computed at $1 \mathrm{KHz}, I_{1}=2 \mathrm{~A}$, $I_{2}=0 \mathrm{~A}, \hat{i}_{1}=0.1 \mathrm{~A}, \hat{i}_{2}=0 \mathrm{~A}$. Both techniques yields identical results for an amplitude of the high frequency current of $5 \%$. Then, as the ratio between the high frequency current and the bias current increases, the iron losses computed in the time domain are higher than the losses computed in the small-signal simulations. This is due to the fact that in the small-signal simulation, the increase of losses due to the distortion of field caused by high frequency current is not completely modeled. The results of Tab. II can be useful to manage the trade off between the required accuracy and the computational cost of the simulation.

TABLE II

IRON LOSSES DUE TO THE EDDY CURRENTS IN THE LAMINATION FOR DifFERENT VALUES OF HIGH FREQUENCY CURRENT. TIME DOMAIN AND SMALL-SIGNAL SIMULATIONS.

\begin{tabular}{lccc}
\hline$\hat{i}_{1} / I_{1}$ & $\hat{i}_{1}$ & $\begin{array}{c}P_{\text {lam-TD }} \\
\text { (p.u.) }\end{array}$ & $\begin{array}{c}P_{\text {lam-SS }} \\
\text { (p.u.) }\end{array}$ \\
$5 \%$ & $(\mathrm{~A})$ & 1 & 1 \\
$10 \%$ & 0.1 & 4.196 & 4.156 \\
$20 \%$ & 0.4 & 16.471 & 15.961 \\
$30 \%$ & 0.6 & 42.235 & 35.922 \\
$40 \%$ & 0.8 & 88.235 & 63.863 \\
\hline
\end{tabular}

Fig. 5 and Fig. 6 show an example of the voltage and current waveforms at $1000 \mathrm{~Hz}$ during two time domain simulations. The bias current and voltage have been removed from the graph, i.e. only the high frequency components are shown. In the first case, Fig. 5, the ratio between high frequency and bias current is $\hat{i}_{1} / I_{1}=0.05$. In this case the voltage is almost sinusoidal, confirming the suitability of the small-signal technique.

In the second case, Fig. 6, the currents ratio is $\hat{i}_{1} / I_{1}=0.4$. As can be noted, the voltage is very distorted resulting in a larger approximation for the small-signal technique.

2) global quantities computation: in this part the main global quantities such as flux linkage, losses and terminal voltage are reported with some maps of the magnetic flux density and flux lines.

Fig. 7 shows a detail of the geometry when the two port mutual inductor is working in a specific operating condition. Fig. 7(a) shows the magnetic flux density in the iron core produced by $I_{1}=1 \mathrm{~A}, I_{2}=0 \mathrm{~A}$, and Fig. $7(\mathrm{~b})$ shows the magnetic flux lines at $10 \mathrm{kHz}$ when $i_{1}=0.05 \mathrm{~A}$, $i_{2}=0 \mathrm{~A}$ are applied. It is worth noticing that magnetic flux lines tends to follow the path where the reluctance is lower. Hence, the magnetic flux lines are more dense towards the upper corner of the iron core.

The two port mutual inductor has been simulated in different operating conditions in order to achieve the flux linkage, the supplying voltage as well as the iron losses due to the eddy currents. In particular, the 3D-TD, 3D-SS, 2DTD, 2D-SS simulations have been performed considering 


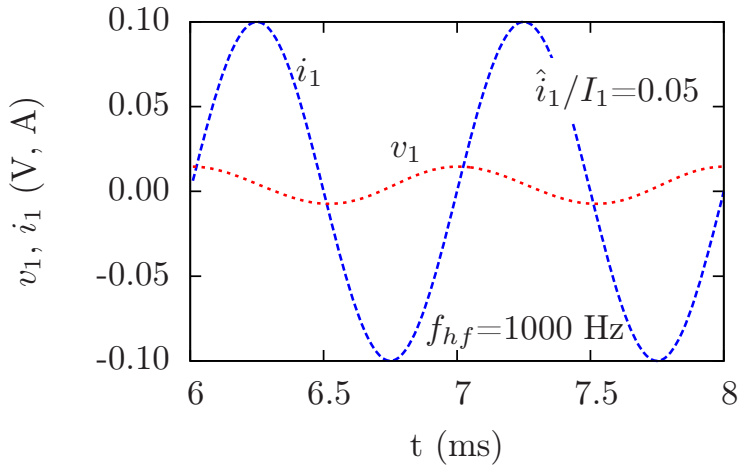

(a) High frequency voltage and current waveforms.

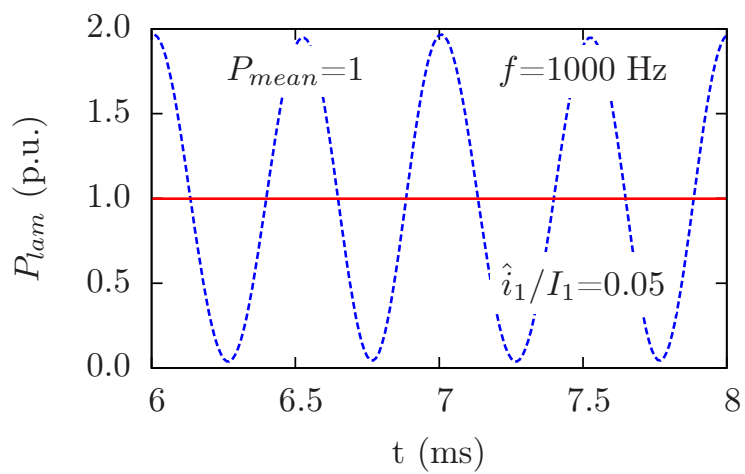

(b) Iron losses due to the eddy currents in the lamination.

Fig. 5. The two port mutual inductor is supplied with bias currents $I_{1}=2 \mathrm{~A}, I_{2}=0 \mathrm{~A}$ and superimposed sinusoidal high frequency currents $\hat{i}_{1}=0.1 \mathrm{~A}, \hat{i}_{2}=0 \mathrm{~A}$ at $1000 \mathrm{~Hz} . i_{1} / I_{1}=0.05$. 2D-TD simulation.

different bias currents $I_{1}=0 \mathrm{~A}, 1 \mathrm{~A}, 2 \mathrm{~A}$, and different injection frequencies in a range between 0 and $10 \mathrm{kHz}$ as well as an injection current $i_{1}=0.05 \mathrm{~A}$. Both $I_{2}$ and $i_{2}$ are always imposed equal to zero. The results are expressed in p.u. considering the results of the 2D-SS simulations.

Fig. 8 shows the flux linkage $\lambda_{1}$ by the primary winding vs the injection frequency. The flux linkage decrease with the injection frequency due to the skin effects present in the iron lamination. When $I_{1}$ increases the flux linkage decreases and tends to be linearized due to the high level of saturation.

In Fig. 9 the supplying voltage vs the injection frequency is shown. Since the injection current is maintained constant, the voltage has to increase according to (6). When the bias current $I_{1}$ increases, the trend of the voltage is more linear due to the saturation.

Fig. 10 depicts the iron losses $P_{l a m}$ in a lamination vs the injection frequency. As in Fig. 9, the iron losses due to the eddy currents increases with the injection frequency because they depend on the square of the frequency. The 0 A curve becomes flatter due to the penetration depth which decreases with the frequency. The $1 \mathrm{~A}$ and $2 \mathrm{~A}$ curves are more linear due to the saturation and exhibit lower values.

For all the computed quantities, a very good agreement among the different simulation techniques can be noted.

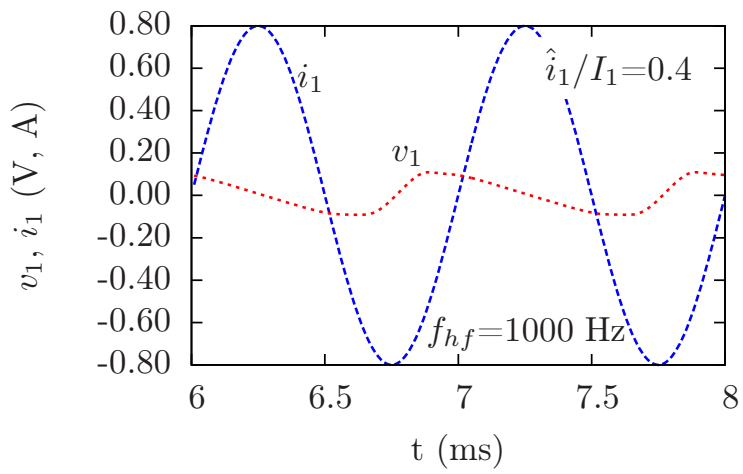

(a) High frequency voltage and current waveforms.

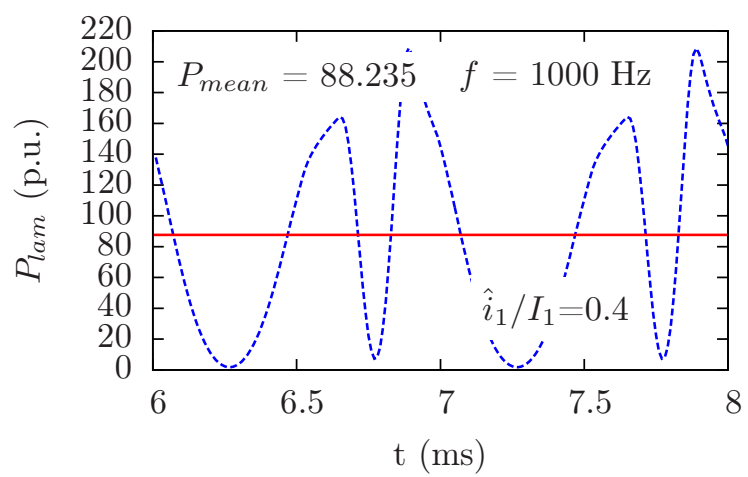

(b) Iron losses due to the eddy currents in the lamination.

Fig. 6. The two port mutual inductor is supplied with a bias current $I_{1}=2 \mathrm{~A}, I_{2}=0 \mathrm{~A}$ and a superimposed sinusoidal high frequency current $\hat{i}_{1}=0.8 \mathrm{~A}, \hat{i}_{2}=0 \mathrm{~A}$ at $1000 \mathrm{~Hz} . i_{1} / I_{1}=0.4$. 2D-TD simulation.

3) Computational costs: As regards the time and memory required by simulations, the results are obtained in the following conditions: the bias currents $I_{1}=1 \mathrm{~A}$ and $I_{2}=0 \mathrm{~A}$; the injection currents $\hat{i}_{1}=0.05 \mathrm{~A}$ and $\hat{i}_{2}=0.05 \mathrm{~A}$; the injection frequency $f=1 \mathrm{kHz}$. The simulations have been carried out on the same workstation. The results are expressed in p.u. with respect the 2D-SS values.

Tab. III reports the time and the memory required by the simulations listed above. As can be noticed, the time taken for the time domain simulations (2D-TD and 3D$\mathrm{TD}$ ) is really long. It mainly depends on the number of the chosen time steps per period and the number of considered periods. For good results at least 100 time steps per period and 8 periods are required. This is due to the fact that for higher frequencies $(8-10 \mathrm{kHz})$ the transient phenomena need more periods then lower frequencies $(1 \mathrm{kHz})$ in order to extinguish. Moreover, 3D simulations (3D-SS and 3DTD) employ more RAM memory due to the higher number of elements in the mesh. In fact, the number of elements in the 3D model is 67231 , while it is 4483 in the $2 \mathrm{D}$ model. Furthermore, time domain simulation (3D-TD and 2D-TD) require more storage memory because the field results have to be saved for each time step. Therefore 2D-SS technique is particularly advantageous because it allows to achieve very accurate results in a shorter time with respect to time domain simulations. 


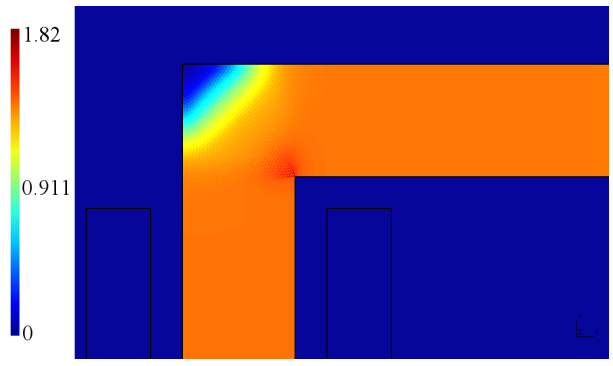

(a) Magnetic flux density ( $\mathrm{T})$ produced by $I_{1}=1 \mathrm{~A}, I_{2}=0 \mathrm{~A}$.

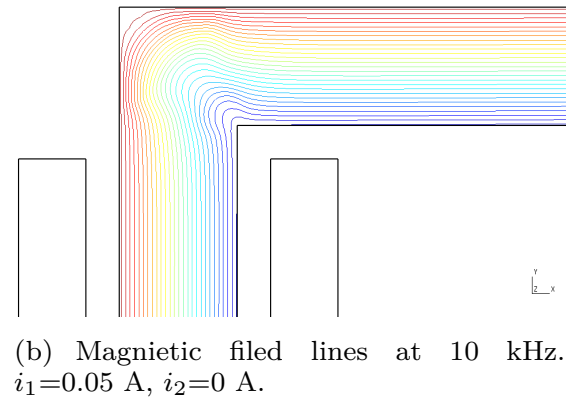

Fig. 7. Magnetic flux maps in a specific working point. Detail of the geometry.

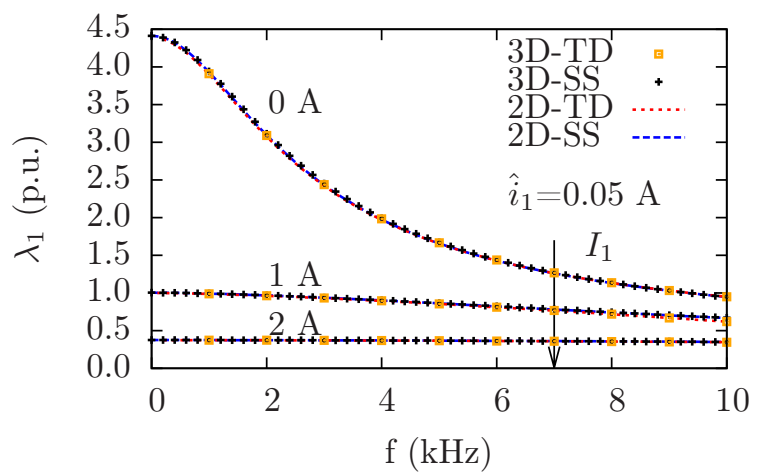

Fig. 8. Flux linkage of the primary coil vs injection frequency considering different values of the bias current $I_{1} .2 \mathrm{D}$ and $3 \mathrm{D}$ simulations using small-signal and time domain techniques are considered.

\section{B. Computation and comparison of incremental parame- ters}

In this section, the computed incremental parameters are shown and compared. All the computational techniques, i.e. 3D-TD, 3D-SS, 2D-TD, 2D-SS, have been considered. The simulations have been performed considering bias currents $I_{1}=0 \mathrm{~A}, 1 \mathrm{~A}, 2 \mathrm{~A}$, and $I_{2}=0 \mathrm{~A}$ on which $i_{1}=i_{2}=0.05 \mathrm{~A}$ are superimposed and different injection frequencies.

Since the turn number of the windings is the same, i.e. $N_{1}=N_{2}$, and the leakage flux is very low, the following relation hold for the parameters according to (10): $\dot{z}_{11}=-\dot{z}_{12}=-\dot{z}_{21}=\dot{z}_{22}=\dot{z}_{h f}$. For the sake of simplicity, the results are thus shown considering only $\dot{z}_{11}=\dot{z}_{h f}$ and its imaginary and real components named $l_{h f}$ and $r_{h f}$.

Fig. 11 shows the incremental parameters vs the in-

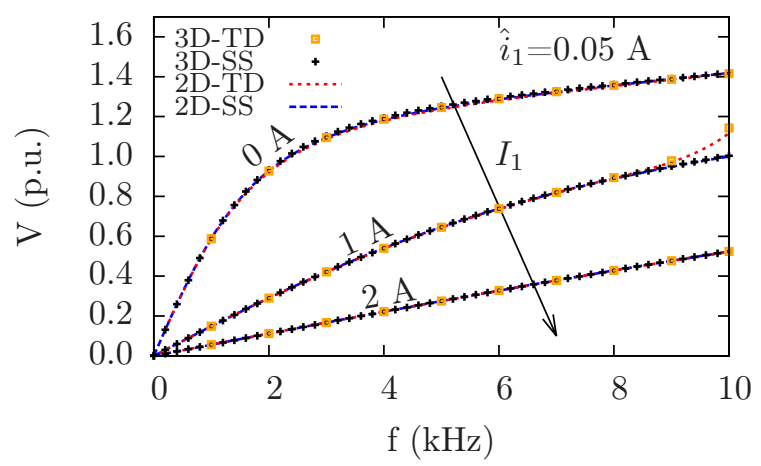

Fig. 9. Voltage on the primary coil vs injection frequency considering different values of the bias current $I_{1} .2 \mathrm{D}$ and $3 \mathrm{D}$ simulations using small-signal and time domain techniques are considered.

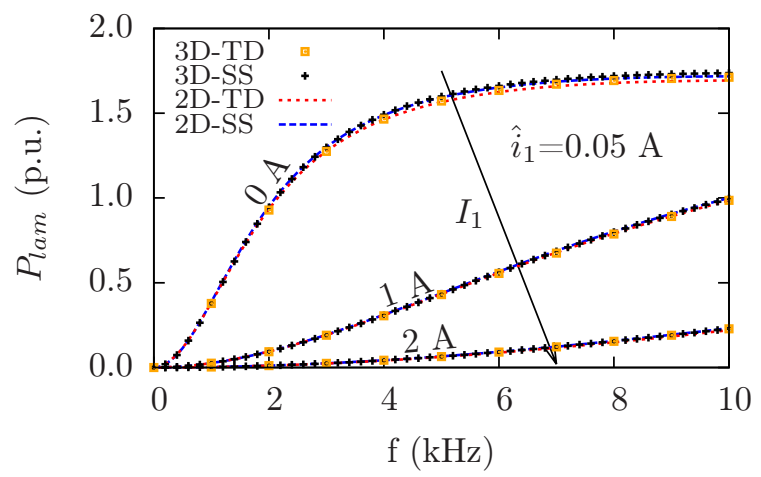

Fig. 10. Lamination losses due to the eddy current vs injection frequency considering different values of the bias current $I_{1} .2 \mathrm{D}$ and 3D simulations using small-signal and time domain techniques are considered.

jection frequency. Fig. 11(a) depicts the incremental inductance that has the same trend as the flux linkage in Fig. 8. The inductive parameters have the same trend as the flux-linkage. Fig. 11(b) shows the incremental resistive parameter which have the same trend of the iron losses in Fig. 10. This aspect can be justified considering $P_{l a m}$ which increase with the frequency powered square. Since the winding resistance can be neglected because only a lamination thickness is considered in the models, the total power at the primary terminals can be thus written as $P_{l a m}=r_{h f} i_{1}^{2}$. Therefore, the variation of active power at the primary terminals mostly depends on the iron losses due to the eddy currents. Consequently, if the iron losses increase, the incremental resistive parameters increase as well.

A good agreement has been found among the results obtained with the four simulations techniques.

\section{Conclusions}

In this paper, the small-signal finite element simulation technique has been validated. Both $2 \mathrm{D}$ and $3 \mathrm{D}$ formulations have been considered and the proposed technique has been compared with time domain formulations. 
TABLE III

TIME AND MEMORY REQUIREMENTS

\begin{tabular}{lccc}
\hline & time & RAM memory & $\begin{array}{c}\text { storage memory } \\
\text { (p.u.) }\end{array}$ \\
(p.u.) & (p.u.) \\
3D-TD & 33470 & 19 & 222 \\
3D-SS & 90 & 15 & 17 \\
2D-TD & 1531 & 2 & 34 \\
2D-SS & 1 & 1 & 1 \\
\hline
\end{tabular}

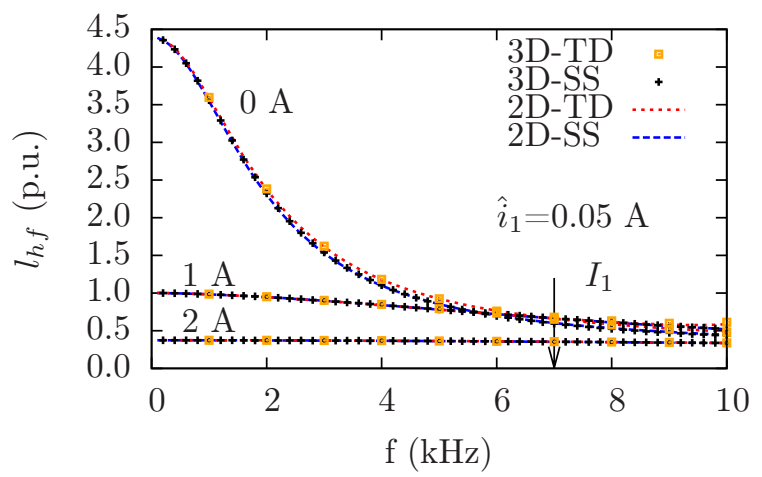

(a) Incremental inductance.

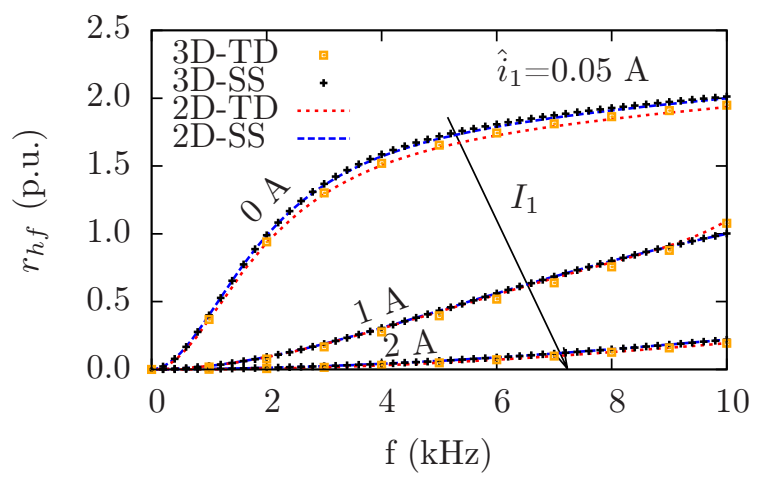

(b) Incremental resistance.

Fig. 11. Incremental parameters vs injection frequency for different values of the bias current $I_{1}$ and considering 3D-TD, 3D-SS, 2D-TD and 2D-SS simulations. High frequency injection current $\hat{i}_{1}=0.05 \mathrm{~A}$.

Additionally, eddy currents in the ferromagnetic material has been taken into account in the various models. In the $2 \mathrm{D}$ model, the eddy current have been implemented through an homogenization technique while, in the 3D model, they have been explicitly modeled and computed.

A two port mutual inductor has been considered as an application example. The results found through time domain simulations and small-signal technique with frozen permeability are in very good agreement. Moreover, it is shown that using the $2 \mathrm{D}$ model and adopting the small-signal technique allows to reduce significantly the computational time and the model complexity maintaining almost the same accuracy, highlighting the benefits of such an analysis technique.

\section{REFERENCES}

[1] A. Bossavit, Computational Electromagnetism. Variational Formulations, Edge Elements, Complementarity. Academic Press,
1998.

[2] N. S. JOAO PEDRO A. BASTOS, Electromagnetic modeling by Finite Element Methods. marchel dekker, 2003.

[3] P. Dular, J. Gyselinck, C. Geuzaine, N. Sadowski, and J. P. A. Bastos, "A 3-d magnetic vector potential formulation taking eddy currents in lamination stacks into account," IEEE Transactions on Magnetics, vol. 39, no. 3, pp. 1424-1427, May 2003.

[4] H. V. Jorks, E. Gjonaj, T. Weiland, and O. Magdun, "Threedimensional simulations of an induction motor including eddy current effects in core laminations," IET Science, Measurement Technology, vol. 6, no. 5, pp. 344-349, September 2012.

[5] P. Handgruber, A. Stermecki, O. Bíró, A. Belahcen, and E. Dlala, "Three-dimensional eddy-current analysis in steel laminations of electrical machines as a contribution for improved iron loss modeling," IEEE Transactions on Industry Applications, vol. 49, no. 5, pp. 2044-2052, Sept 2013.

[6] C. V. S. R. Gyselinck, Johan. Geuzaine, "Considering laminated cores and eddy currents in $2 \mathrm{~d}$ and $3 \mathrm{~d}$ finite element simulation of electrical machines," 18th Conference on the Computation of Electromagnetic Fields (COMPUMAG2011), p. 2, 07 2011, event date: July 12-15, 2011. [Online]. Available: http://hdl.handle.net/2268/92146

[7] L. Alberti, N. Bianchi, M. Morandin, and J. Gyselinck, "Finiteelement analysis of electrical machines for sensorless drives with high-frequency signal injection," Industry Applications, IEEE Transactions on, vol. 50, no. 3, pp. 1871-1879, May 2014.

[8] L. Alberti, N. Bianchi, and S. Bolognani, "High frequency d-q model of synchronous machines for sensorless control," Industry Applications, IEEE Transactions on, vol. PP, no. 99, pp. 1-1, 2015.

[9] D. Mingardi, N. Bianchi, and L. Alberti, "Analysis and test of the sensorless capability of induction motors with created saliency," IEEE Transactions on Industry Applications, vol. PP, no. 99, pp. 1-1, 2016.

[10] S. C. Yang and R. D. Lorenz, "Comparison of resistancebased and inductance-based self-sensing controls for surface permanent-magnet machines using high-frequency signal injection," IEEE Transactions on Industry Applications, vol. 48, no. 3, pp. 977-986, May 2012.

[11] J. Gyselinck, R. Sabariego, and P. Dular, "A nonlinear timedomain homogenization technique for laminated iron cores in three-dimensional finite-element models," IEEE Transactions on Magnetics, vol. 42, no. 4, pp. 763-766, April 2006.

[12] N. Bianchi and S. Bolognani, "Magnetic models of saturated interior permanent magnet motors based on finite element analysis," in Industry Applications Conference, 1998. Thirty-Third IAS Annual Meeting. The 1998 IEEE, vol. 1, Oct 1998, pp. 2734 vol.1.

[13] J. A. Walker, D. G. Dorrell, and C. Cossar, "Flux-linkage calculation in permanent-magnet motors using the frozen permeabilities method," IEEE Transactions on Magnetics, vol. 41, no. 10 , pp. 3946-3948, Oct 2005.

[14] J. K. Tangudu, T. M. Jahns, A. M. El-Refaie, and Z. Q. Zhu, "Segregation of torque components in fractional-slot concentrated-winding interior pm machines using frozen permeability," in 2009 IEEE Energy Conversion Congress and Exposition, Sept 2009, pp. 3814-3821.

[15] P. Dular, F. Henrotte, and W. Legros, "A general and natural method to define circuit relations associated with magnetic vector potential formulations," IEEE Transactions on Magnetics, vol. 35, no. 3, pp. 1630-1633, May 1999.

[16] J. Gyselinck, P. Dular, N. Sadowski, J. Leite, and J. Bastos, "Incorporation of a jiles-atherton vector hysteresis model in 2d fe magnetic field computations: Application of the newtonraphson method," COMPEL - The international journal for computation and mathematics in electrical and electronic engineering, vol. 23, no. 3, pp. 685-693, 2004. [Online]. Available: http://dx.doi.org/10.1108/03321640410540601 